International Journal of Modern Physics A

(C) World Scientific Publishing Company

\title{
CHARGED LEPTON-NUCLEUS INELASTIC SCATTERING AT HIGH ENERGIES
}

\author{
K. S. KUZMIN \\ BLTP, Joint Institute for Nuclear Research, Dubna, Moscow Region, Russia \\ Institute for Theoretical and Experimental Physics, Moscow, Russia \\ kkuzmin@thsun1.jinr.ru \\ K. S. LOKHTIN and S. I. SINEGOVSKY \\ Department of Theoretical Physics, Irkutsk State U., Irkutsk, Russia \\ sinegovsky@api.isu.ru
}

Received (Day Month Year)

Revised (Day Month Year)

\begin{abstract}
The composite model is constructed to describe inelastic high-energy scattering of muons and taus in standard rock. It involves photonuclear interactions at low $Q^{2}$ as well as moderate $Q^{2}$ processes and the deep inelastic scattering (DIS). In the DIS region the neutral current contribution is taken into consideration. Approximation formulas both for the muons and tau energy loss in standard rock are presented for wide energy range.
\end{abstract}

Keywords: muon inelastic scattering off nuclei; tau-lepton energy loss

\section{Introduction}

The muon inelastic scattering off nuclei contributes noticeably to the energy loss of cosmic rays muons. The influence of this interaction on the shape of ultra-high energy muon spectra at the great depth of a rock/water is still unknown in detail. The tau-lepton energy loss is of interest in view of ability of atmospheric or extraterrestrial muon neutrinos to transform to tau neutrinos which may produce taus in $\nu N$-interactions. The validity of the well known model of photonuclear interactions 1, employed over a long period in computations of the cosmic rays muon energy loss and the muon depth-intensity relation (e. g. 21344), is not apparent at very high energies. Certainly low $Q^{2}$ processes give dominant contribution to the scattering, nevertheless the large $Q^{2}$ may contribute significantly at very high energies. Recently the high momentum transfer in high-energy lepton-nucleon interactions was taken into account by diverse ways 15617 . Unlike 517 in Ref. 66 $Z^{0}$ exchange processes were taken into consideration but there only scattering of muons was involved. Present computation is close to the former differing however from that in some points: i) very low $Q^{2}$ are considered separately from moderate $Q^{2}$; ii) calculations are performed for wider range of lepton energies, $E=10^{3}-10^{9} \mathrm{GeV}$; 
iii) both muon and tau energy loss spectra are calculated.

\section{The model}

The three-component model (3-model) for inelastic interactions of high-energy muons and taus with nuclei involves photonuclear interactions at low momentum transfer squared as well as moderate $Q^{2}$ processes and the deep inelastic scattering (DIS). For low $Q^{2}\left(<0.1 \mathrm{GeV}^{2}\right)$ the structure function (SF) parametrization 1 based on the generalized vector-meson dominance model was used. The Regge based model CKMT 8 was applied for moderate values of $Q^{2}, 0.1<Q^{2}<5 \mathrm{GeV}^{2}$. In the DIS region the electroweak nucleon SFs and lepton-nucleus cross sections are computed with the CTEQ6 $\frac{9}{9}$ and MRST ${ }^{10}$ sets of parton distributions. Linear fits for the nucleon SF are used both in $0.09<Q^{2}<0.1 \mathrm{GeV}^{2}$ and $5<Q^{2}<6 \mathrm{GeV}^{2}$ ranges. Also considered is the two-component version (2-model) that consists of the CKMT model and the DIS calculations. For the scattering off nuclei effects of the nucleon shadowing, anti-shadowing as well as EMC effect are taken into account according to Ref. [6] (see Ref. 11] for details). The energy loss spectra for lepton passing a substance with nuclear weight $A$ can be derived from the differential cross-section:

$$
\frac{N_{A}}{A} y \frac{d \sigma^{\ell A}}{d y}=\frac{N_{A}}{A} y \int_{Q_{\min }^{2}}^{Q_{\max }^{2}} d Q^{2} \frac{d^{2} \sigma^{\ell A}}{d Q^{2} d y}, \quad y=\frac{E-E^{\prime}}{E}=\frac{\nu}{E} .
$$

The energy loss due to lepton-nucleus interactions is defined with the integral

$$
b_{N}^{(\ell)}(E) \equiv-\frac{1}{E} \frac{d E}{d h}=\frac{N_{A}}{A} \int_{y_{\min }}^{y_{\max }} y \frac{d \sigma^{\ell A}}{d y} d y .
$$

\section{Results}

Figs. 11 2 show the energy loss spectra of muons (the left panel) and of taus in standard rock $(A=22)$ calculated for lepton energies $E=10^{5} \mathrm{GeV}$ and $E=10^{8}$ $\mathrm{GeV}$. The result of the 2-model (solid line) differs visibly from that of the 3-model (dash-dotted) only in small $y$ range. Dotted lines show the differential cross-section $d \sigma^{(\mu, \tau) A} / d y$ calculated with the approximation for the "nonperturbative part" 7, dashed lines present our calculation with the parametrization 1 . One can conclude that for not too large energies $\left(E<10^{5} \mathrm{GeV}\right)$ all these spectra differ significantly only at low $y$. As the lepton energy increases distinctions between the 2(3)-model energy loss spectra and those of Ref. 1 concern the range of $y>0.1$ that gives dominant contribution to the lepton energy loss.

Predictions for the energy loss of muons and taus in standard rock are shown in the Fig. 3. Circles here show calculations with the 3-model version in which nuclear effects for low- $Q^{2}$ component (BB) were taken into account after Ref. [6] instead of those in Ref.11. Apparently the difference between the 2-model and 3-model appears because of nuclear effects, the anti-shadowing, EMC-effect and Fermi motion, which were not taken into consideration in Ref. 1]. 

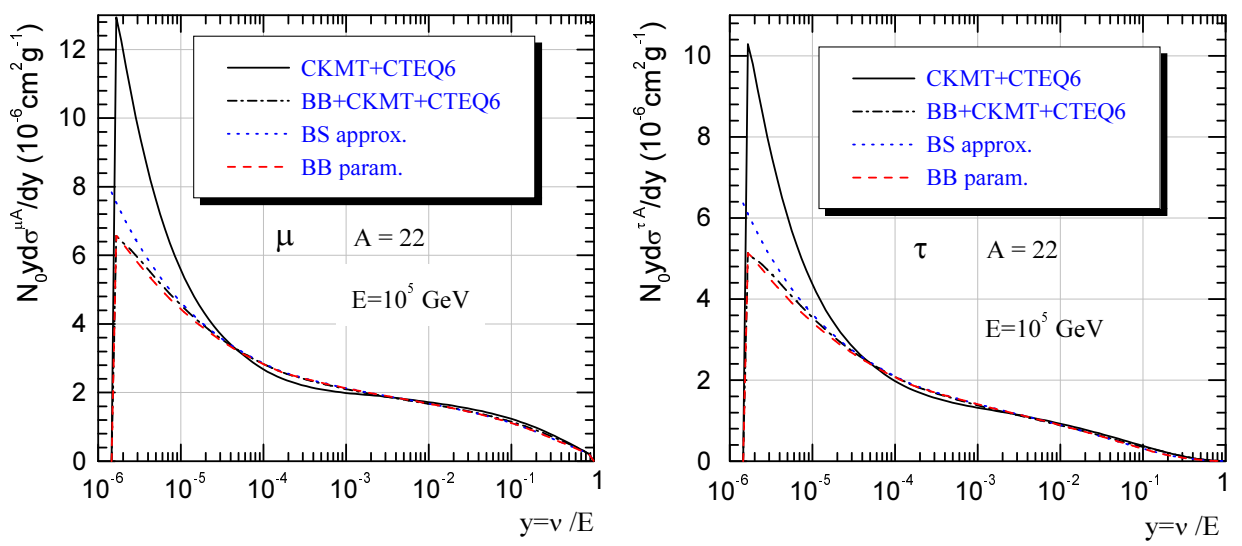

Fig. 1. Spectra of the lepton energy loss in standard rock at $E=10^{5} \mathrm{GeV}$.
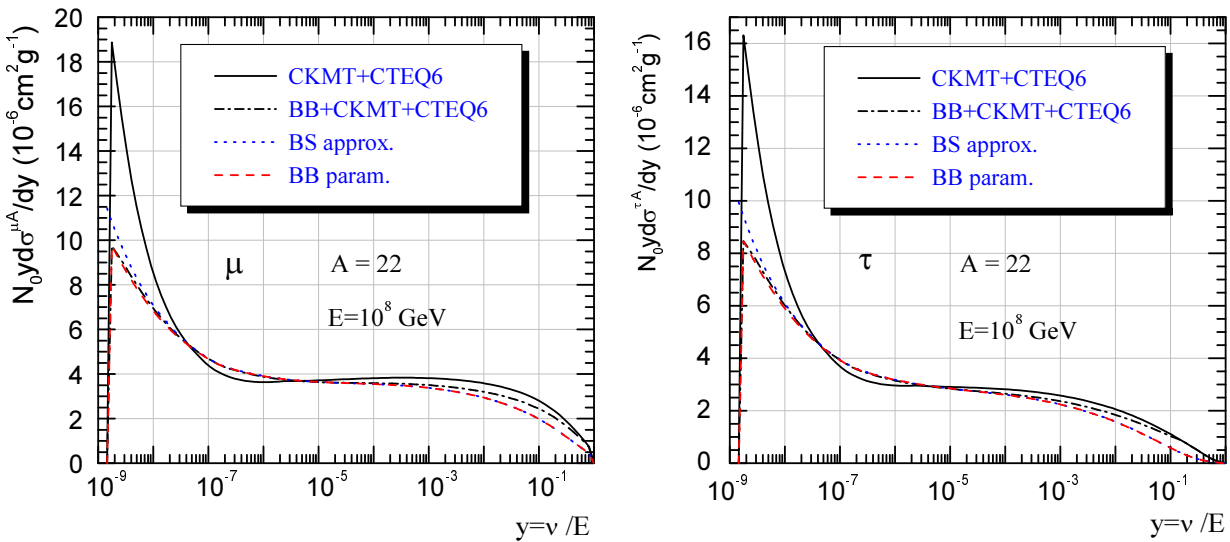

Fig. 2. Spectra of the lepton energy loss in standard rock at $E=10^{8} \mathrm{GeV}$.

The Fig. 4 shows this work calculation (2-model) of the muon energy loss in standard rock (the median solid line) compared to the recent one of Ref. 7 (upper line) (see also Table 1) and to the old result of Ref. 1 (dashed).

The energy dependence of calculated muon and tau energy loss in standard rock can be approximated with the formula $(\ell=\mu, \tau)$ :

$$
\begin{aligned}
& b_{N}^{(\ell)}(E)=\left(c_{0}+c_{1} \eta+c_{2} \eta^{2}+c_{3} \eta^{3}+c_{4} \eta^{4}\right) \cdot 10^{-6} \mathrm{~cm}^{2} / \mathrm{g}, \eta=\lg (E / 1 \mathrm{GeV}) ; \quad(3) \\
& \mu: \quad c_{0}=0.98711, c_{1}=-0.56840, c_{2}=0.17677, c_{3}=-0.02114, c_{4}=0.00112 ; \\
& \tau: \quad c_{0}=0.33247, c_{1}=-0.22283, c_{2}=0.06811, c_{3}=-0.00873, c_{4}=0.00048 .
\end{aligned}
$$

In the second column of the Table 1 the 2-model calculation of the muon and tau energy loss in standard rock and that of the 3-model (in brackets) are presented 

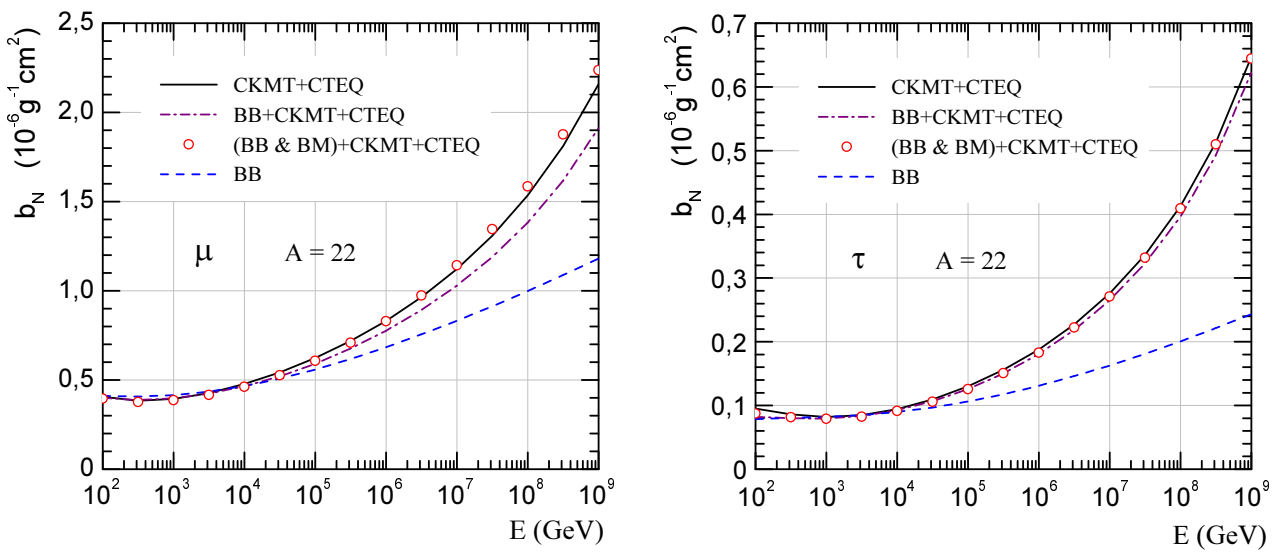

Fig. 3. Muon and tau energy loss in standard rock $(A=22)$ computed with the 2- and 3-model.

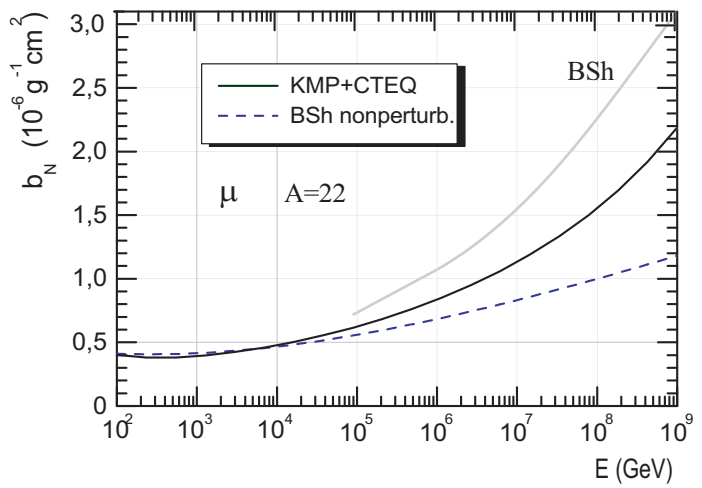

Fig. 4. Comparison of muon energy loss calculations. The upper line presents the result of Ref.7

Table 1. The muon and tau energy loss in standard rock.

\begin{tabular}{|c|c|c|c|c|c|}
\hline $\begin{array}{c}E, \\
\mathrm{GeV}\end{array}$ & \multicolumn{2}{|c|}{ This work } & \multicolumn{3}{|c|}{$\overline{b_{N}^{(\ell)}(E), 10^{-6} \cdot \mathrm{cm}^{2} g^{-1}}$} \\
\hline \multicolumn{6}{|c|}{ Muon } \\
\hline $10^{5}$ & 0.62 & (0.59) & 0.60 & 0.68 & 0.70 \\
\hline $10^{6}$ & 0.82 & $(0.78)$ & 0.80 & 0.90 & 1.08 \\
\hline $10^{8}$ & 1.53 & (1.38) & 1.50 & - & 2.25 \\
\hline $10^{9}$ & 2.16 & $(1.91)$ & 2.15 & - & 3.10 \\
\hline \multicolumn{6}{|c|}{ Tau } \\
\hline $10^{5}$ & 0.13 & $(0.13)$ & 0.12 & - & 0.12 \\
\hline $10^{6}$ & 0.19 & $(0.18)$ & 0.18 & - & 0.18 \\
\hline $10^{8}$ & 0.41 & $(0.40)$ & 0.40 & - & 0.44 \\
\hline $10^{9}$ & 0.65 & $(0.62)$ & 0.60 & - & 0.66 \\
\hline
\end{tabular}


along with recent predictions 5167 . One can see that present calculations of taulepton energy loss in standard rock are close to the results of other authors. As concerns to muons, this work result for $b_{N}^{(\mu)}$ differs apparently from that of Ref. [7]

\section{Summary}

One can conclude: (i) The low $Q^{2}$ contribution to spectra of the muon and tau energy loss predicted by BB is close to the CKMT one but the small-y region; (ii) The nucleon anti-shadowing and EMC effect influence visibly on muon energy loss; (iii) No apparent contribution of the neutral current to energy loss spectra of muons and taus in standard rock was found up to $10^{9} \mathrm{GeV}$; (iv) There is noticeable discrepancy between this work prediction for high-energy behavior of the muon

energy loss, $b_{N}^{(\mu)}(E)$, and that of Ref. [7] likely due to diverse ways in considering of high $Q^{2}$ processes and nuclear effects.

\section{Acknowledgements}

S.I.S. thanks the $19^{\text {th }}$ ECRS Organizing Committee for the financial support, Edgar Bugaev and Vadim Naumov for valuable discussions.

\section{References}

1. L. B. Bezrukov, E. V. Bugaev, Sov. J. Nucl. Phys. 33, 635 (1981).

2. E. V. Bugaev, A. Misaki, V. A. Naumov et al., Phys. Rev. D58, 054001 (1998); hep-ph/9803488

3. T. S. Sinegovskaya, S.I. Sinegovsky, Phys. Rev. D63, 096004 (2001); hep-ph/0007234

4. A. Misaki, T. S. Sinegovskaya, S. I. Sinegovsky, N. Takahashi, J. Phys. G: Nucl. Part. Phys. 29, 387 (2003); hep-ph/0302183

5. S. I. Dutta, M. H. Reno, I. Sarcevic, D. Seckel, Phys. Rev. 63, 094020 (2001).

6. A. V. Butkevich, S. P. Mikheyev, Zh. Eksp. Teor. Fiz. 122, 17 (2002); hep-ph/0109060

7. E. V. Bugaev, Yu. V. Shlepin, Phys. Rev. D67, 034027 (2003).

8. A.Capella, A. B. Kaidalov, C. Merino, J.Tran Thanh Van, Phys. Lett. B337, 358 (1994); hep-ph/9405338

A. B. Kaidalov, C. Merino, D. Pertermann, Eur. Phys. J. C20, 301 (2001).

9. J. Pumplin et al., JHEP 0207, 012 (2002); hep-ph/0201195

10. A.D. Martin, R.G. Roberts, W.J. Stirling, R. S. Thorne, Eur. Phys. J. C23, 73 (2002).

11. M. Arneodo, Phys. Rept. 240, 301 (1994). 